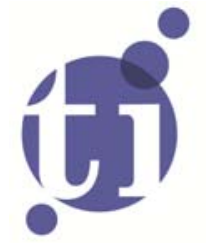

\title{
Cooperative Games on Accessible Union Stable Systems
}

\author{
Encarnación Algabal \\ René van den Brink² \\ Chris Dietz²
}

' Escuela Técnica Superior de Ingenierá, Sevilla, Spain;

2 Faculty of Economics and Business Administration, VU University Amsterdam, and Tinbergen Institute, The Netherlands. 
Tinbergen Institute is the graduate school and research institute in economics of Erasmus University Rotterdam, the University of Amsterdam and VU University Amsterdam.

More TI discussion papers can be downloaded at http://www.tinbergen.nl

Tinbergen Institute has two locations:

Tinbergen Institute Amsterdam

Gustav Mahlerplein 117

1082 MS Amsterdam

The Netherlands

Tel.: +31(0)205251600

Tinbergen Institute Rotterdam

Burg. Oudlaan 50

3062 PA Rotterdam

The Netherlands

Tel.: +31(0)10 4088900

Fax: $+31(0) 104089031$

Duisenberg school of finance is a collaboration of the Dutch financial sector and universities, with the ambition to support innovative research and offer top quality academic education in core areas of finance.

DSF research papers can be downloaded at: http://www.dsf.nl/

Duisenberg school of finance

Gustav Mahlerplein 117

1082 MS Amsterdam

The Netherlands

Tel.: +31(0)20 5258579 


\title{
Cooperative games on accessible union stable systems ${ }^{1}$
}

\author{
Encarnación Algaba² René van den Brink ${ }^{3}$ Chris Dietz ${ }^{4}$
}

December 17, 2013

\footnotetext{
${ }^{1}$ This research is financially supported by the Netherlands Organization for Scientific Research, NWO grant 400-08-026.

${ }^{2}$ Matemática Aplicada II, Escuela Superior de Ingenieros, Camino de los Descubrimientos, s/n, 41092 Sevilla, Spain. E-mail: ealgaba@us.es.

${ }^{3}$ J.R. van den Brink, Department of Econometrics and Tinbergen Institute, VU University Amsterdam, De Boelelaan 1105, 1081 HV Amsterdam, the Netherlands. E-mail: jrbrink@feweb.vu.nl

${ }^{4}$ Department of Econometrics and Tinbergen Institute, VU University, De Boelelaan 1105, 1081 HV Amsterdam, The Netherlands. E-mail: cdietz@feweb.vu.nl.
} 


\begin{abstract}
Agents participating in different kind of organizations, usually take different positions in some relational structure. The aim of this paper is to introduce a new framework taking into account both communication and hierachical features derived from this participation. In fact, this new set or network structure unifies and generalizes well-known models from the literature, such as communication networks and hierarchies. We introduce and analyze accessible union stable systems where union stability reflects the communication network and accessibility describes the hierarchy. Particular cases of these new structures are the sets of connected coalitions in a communication graph, antimatroids (and therefore also sets of feasible coalitions in permission structures) and augmenting systems which have numerous applications in the literature. We give special attention to the class of cyclefree accessible union stable systems. We also consider cooperative games with restricted cooperation where the set of feasible coalitions is an accessible union stable system, and provide an axiomatization of an extension of the Shapley value to this class of games.
\end{abstract}

Key words: union stable system, accessibility, cooperative TU-game, Shapley value

JEL code: C71

AMS subject classifications: $91 \mathrm{~A} 12$ 


\section{Introduction}

A cooperative game with transferable utility, or simply a TU-game, is a finite set of players and for any subset (coalition) of players a worth representing the total payoff that the coalition can obtain by cooperating. A (single-valued) solution is a function that assigns to every game a payoff vector which components are the individual payoffs to each player. A solution is efficient if it assigns to every game a payoff vector such that the sum of the payoffs is equal to the worth of the 'grand coalition' consisting of all players. One of the most applied efficient solutions for cooperative TU-games is the Shapley value (Shapley, 1953).

In its classical interpretation, a TU-game describes a situation in which every coalition $S$ (i.e subset) of $N$ can be formed and earn its worth. In the literature various restrictions on coalition formation have been developed. Two main forms of restricted cooperation that have been studied are communication restrictions and hierarchies. Myerson (1977) introduced the well-known model of a communication graph game that consists of a TU-game and an undirected (communication) graph where it is assumed that only coalitions that are connected in the communication graph are feasible. A restricted game is defined where the worth of every feasible (i.e. connected) coalition equals its worth in the original game, while the worth of a nonconnected coalition equals the sum of the worths of its maximally connected subsets (also known as components) of the coalition. Further, he showed that the solution that assigns to every communication graph game the Shapley value of the restricted game is the only solution that satisfies component efficiency (meaning that every maximally connected subset of players earns its own worth) and fairness (meaning that deleting a communication link between two players has the same effect on the individual payoffs of these two players). Algaba, Bilbao, Borm and López (2001) generalized this result to games on union stable systems being set systems that satisfy the property that the union of every pair of nondisjoint coalitions is also feasible, a property that is satisfied by the set of connected coalitions of any undirected graph.

A model that studies restrictions in cooperation arising from hierarchies is that of a game with a permission structure. In those games it is assumed that the players are part of a hierarchical organization, where some players might need permission or approval from other players before they are allowed to cooperate. Two approaches to games with a permission structure are considered. In the conjunctive approach as developed in Gilles, Owen and van den Brink (1992) and van den Brink and Gilles (1996), it is assumed that each player needs permission from all its predecessors before it is allowed to cooperate with other players. This implies that a coalition is feasible if and only if for every player in the coalition it holds that all its predecessors belong to the coalition. Alternatively, in the disjunctive approach as developed in Gilles and Owen (1994) and van den Brink (1997), it is assumed that each 
player (except the top-players) needs permission from at least one of its predecessors before it is allowed to cooperate. Consequently, a coalition is feasible if and only if every (nontop) player in the coalition has at least one predecessor who also belongs to the coalition. In Algaba, Bilbao, van den Brink and Jiménez-Losada (2004) it is shown that the sets of feasible coalitions arising from these permission structures are antimatroids being wellknown combinatorial structures representing hierarchies, see Dilworth (1940) and Edelman and Jamison (1985). A set of feasible coalitions is an antimatroid if it contains the empty set, satisfies accessibility (meaning that every nonempty feasible coalition has at least one player that can leave the coalition and the result is a feasible subcoalition) and is union closed (meaning that the union of two feasible coalitions is also feasible).

In the field of restricted cooperation, van den Brink (2012) made clear the distinction between hierarchies and communication networks by showing that the set systems that can be the set of connected coalitions in some undirected graph are exactly those set systems that, besides containing the empty set, satisfy the above mentioned union stability and 2-accessibility (meaning that every feasible coalition with two or more players has at least two players that can leave the coalition such that the remaining set of players is still a feasible coalition). So, compared to communication feasible sets (set systems that can be obtained as the set of connected coalitions in some undirected graph), antimatroids satisfy a stronger union property (since union closedness implies union stability) but a weaker accessibility property (since 2 -accessibility implies accessibility).

The goal of the underlying paper is to get a better understanding of organizations that have hierarchical as well as communication features. Therefore, we study set systems that satisfy the weaker union and accessibility properties, i.e. set systems that contain the empty set and satisfy union stability and accessibility. We call these accessible union stable systems. Obviously, all sets of connected coalitions of some (undirected) communication graph as well as all antimatroids fall into this class. We also show that augmenting systems form a proper subclass of these new structures. After discussing some results on these structures, we give special attention to the subclass of accessible union stable systems that are cycle-free and show that under accessibility and feasibility of the unitary coalitions, cycle-free union stable systems are exactly those that can be obtained as the set of connected coalitions in a cycle-free communication graph. Then, we consider cooperative TU-games with restricted cooperation where the set of feasible coalitions is an accessible union stable system. We consider the solution that assigns to every cooperative game on an accessible union stable system the Shapley value of a restricted game where the worths are generated only by feasible coalitions. Finally, we provide an axiomatization of the Shapley value on the class of games on an accessible union stable system using a balanced contributions axiom, and an axiomatization on the class of cycle-free accessible 
union stable systems using a fairness axiom.

The paper is organized as follows. Section 2 gives some preliminaries. In Section 3 we introduce accessible union stable systems and study some of their properties. In Section 4 we analyze how the property of accessibility influences the basis of these structures. In Section 5 we consider cycle-free accessible union stable systems. In Section 6 we extend TU-games with a set of feasible coalitions that is an accessible union stable system, presenting the Shapley value and providing axiomatizations. Finally, Section 7 contains some concluding remarks.

\section{Preliminaries: Communication and hierarchies in cooperative games}

\subsection{Cooperative TU-games and restricted cooperation}

A situation in which a finite set of players can obtain certain payoffs by cooperation can be described by a cooperative game with transferable utility, or simply a TU-game, being a pair $(N, v)$, where $N \subseteq \mathbb{N}$ is a finite set of players and $v: 2^{N} \rightarrow \mathbb{R}$ is a characteristic function on $N$ satisfying $v(\emptyset)=0$. For any coalition $S \subseteq N, v(S)$ is the worth of coalition $S$, meaning that the members of coalition $S$ can obtain a total payoff of $v(S)$ by agreeing to cooperate. Since we take the player set to be fixed, we denote a TU-game $(N, v)$ just by its characteristic function $v$. We denote the collection of all TU-games on player set $N$ by $\mathcal{G}^{N}$.

A payoff vector of an $n$-player TU-game $v \in \mathcal{G}^{N}$ is an $n$-dimensional vector $x \in \mathbb{R}^{N}$ giving a payoff $x_{i} \in \mathbb{R}$ to any player $i \in N$. A (single-valued) solution for TU-games is a mapping $f$ that assigns to every game $v \in \mathcal{G}^{N}$ a payoff vector $f(v) \in \mathbb{R}^{N}$. One of the most well-known solutions for TU-games is the Shapley value (Shapley (1953)) given by

$$
S h_{i}(v)=\sum_{\substack{S \subseteq N \\ i \in S}} \frac{(|N|-|S|) !(|S|-1) !}{|N| !}(v(S)-v(S \backslash\{i\})) \text { for all } i \in N \text {. }
$$

In a TU-game any subset $S \subseteq N$ is assumed to be able to form a coalition and earn the worth $v(S)$. However, in most economic and political organizations not every set of participants can form a feasible coalition. Therefore, in cooperative game theory models have been developed that take account of restrictions on coalition formation. This is modelled by considering a set of feasible coalitions $\mathcal{F} \subseteq 2^{N}$ that need not contain all subsets of the player set $N$. For a finite set $N$, a set system over $N$ is a pair $(N, \mathcal{F})$ where $\mathcal{F} \subseteq 2^{N}$ is a family of subsets. The sets belonging to $\mathcal{F}$ are called feasible. A triple 
$(N, v, \mathcal{F})$ with $v \in \mathcal{G}^{N}$ and $\mathcal{F} \subseteq 2^{N}$ is a game with restricted cooperation. Again, since we take the player set to be fixed, we denote a game with restricted cooperation $(N, v, \mathcal{F})$ by $(v, \mathcal{F})$.

\subsection{Communication}

One of the most well-known models of restricted cooperation are the games with communication restrictions as introduced in Myerson (1977). In this model a communication network on the set of players in a cooperative game is given, and a coalition $S$ is feasible if and only if the players in $S$ are connected within this communication network. This communication network is represented by an undirected graph on the set of players.

An undirected graph is a pair $(N, L)$ where $N$ is the set of nodes and $L \subseteq\{\{i, j\} \mid i, j \in$ $N, i \neq j\}$ is a collection of subsets of $N$ such that each element of $L$ contains precisely two elements. The elements of $L$ represent bilateral communication links and are refered to as edges or links. Since in this paper the nodes in a graph represent the positions of players in a communication network we refer to the nodes as players. If there is a link between two players we call them neighbours. A sequence of $k$ different players $\left(i_{1}, \ldots, i_{k}\right)$ is a path in $(N, L)$ if $\left\{i_{h}, i_{h+1}\right\} \in L$ for $h=1, \ldots, k-1$. Two distinct players $i$ and $j, i \neq j$, are connected in graph $(N, L)$ if there is a path $\left(i_{1}, \ldots, i_{k}\right)$ with $i_{1}=i$ and $i_{k}=j$. A coalition $S \subseteq N$ is connected in graph $(N, L)$ if every pair of players in $S$ is connected by a path that only contains players from $S$, i.e. for every $i, j \in S, i \neq j$, there is a path $\left(i_{1}, \ldots, i_{k}\right)$ such that $i_{1}=i, i_{k}=j$ and $\left\{i_{1}, \ldots, i_{k}\right\} \subseteq S$. A maximally connected subset of coalition $S$ in $(N, L)$ is called a component of $S$ in that graph, i.e. $T \subseteq S$ is a component of $S$ in $(N, L)$ if and only if (i) $T$ is connected in $(N, L(S))$ and (ii) for every $h \in S \backslash T$ the coalition $T \cup\{h\}$ is not connected in $(N, L(S))$, where $L(S)=\{\{i, j\} \in L \mid\{i, j\} \subseteq S\}$ is the set of links between players in $S$.

A sequence of players $\left(i_{1}, \ldots, i_{k}, i_{1}\right), k \geq 2$, is a cycle in $(N, L)$ if $\left(i_{1}, \ldots, i_{k}\right)$ is a path in $(N, L)$ and $\left\{i_{k}, i_{1}\right\} \in L$. A graph $(N, L)$ is cycle-free when it does not contain any cycle.

Example 2.1 Consider the communication graph $(N, L)$ on $N=\{1, \ldots, 5\}$ given by $L=\{\{1,2\},\{1,3\},\{2,4\},\{3,4\},\{4,5\}\}$, see Figure 1 . Players 1 and 5 are connected by two paths: $(1,2,4,5)$ and $(1,3,4,5)$. Coalition $\{1,4,5\}$ has two components: $\{1\}$ and $\{4,5\}$. This communication graph has a cycle $(1,2,4,3,1)$.

A triple $(N, v, L)$ with $(N, v)$ a TU-game and $(N, L)$ an undirected communication graph is called a communication graph game. Since, again we take the player set to be fixed, 


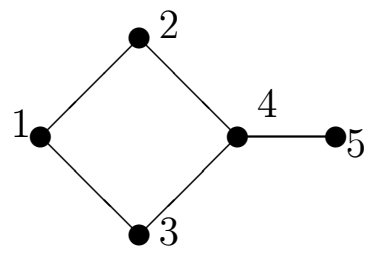

Figure 1: Communication graph $(N, L)$ of Example 2.1

we denote a communication graph game $(N, v, L)$ just by $(v, L)$. In the communication graph game $(v, L)$ players can cooperate if and only if they are able to communicate with each other, i.e. a coalition $S$ is feasible if and only if it is connected in $(N, L)$. In other words, the set of feasible coalitions in a communication graph game $(N, v, L)$ is the set of coalitions $\mathcal{F}_{L} \subseteq 2^{N}$ given by

$$
\mathcal{F}_{L}=\{S \subseteq N \mid S \text { is connected in }(N, L)\}
$$

We refer to this set as the communication feasible set of communication graph $(N, L)$. Myerson (1977) introduced the restricted game of a communication graph game $(v, L)$ as the TU-game $v_{L}$ in which every feasible coalition $S$ can earn its worth $v(S)$. Whenever $S$ is not feasible it can earn the sum of the worths of its components in $(N, L)$. Denoting the set of components of $S \subseteq N$ in $(N, L)$ by $C_{L}(S)$, the restricted game $v_{L}$ corresponding to communication graph game $(v, L)$ is given by ${ }^{1}$

$$
v_{L}(S)=\sum_{T \in C_{L}(S)} v(T) \text { for all } S \subseteq N
$$

The solution given by Myerson (1977) is obtained by taking for every communication graph game the Shapley value of the corresponding restricted game, a solution that was later named the Myerson value $\mu$ for communication graph games, i.e. $\mu(v, L)=\operatorname{Sh}\left(v_{L}\right)$.

\subsection{Hierarchies}

A model that studies restrictions in coalition formation arising from hierarchies is that of a game with a permission structure. In those games it is assumed that players are part of a hierarchical organization in which there are players that need permission or approval from certain other players before they are allowed to cooperate. In this case, for a finite set of players $N$ such a hierarchical organization is represented by an irreflexive directed graph $(N, D)$ with $D \subseteq N \times N$ such that $(i, i) \notin D$ for all $i \in N$, referred to as a

\footnotetext{
${ }^{1}$ Note that $C_{L}(S)$ is a partition of $S$.
} 
permission structure on $N$. Since, again we take the player set to be fixed, we denote a permission structure $(N, D)$ just by its binary relation $D$. The directed links $(i, j) \in D$ are called arcs. The players in $F_{D}(i):=\{j \in N \mid(i, j) \in D\}$ are called the successors or followers of player $i$, while the players in $P_{D}(i):=\{j \in N \mid(j, i) \in D\}$ are called the predecessors of $i$. A sequence of different players $\left(i_{1}, \ldots, i_{k}\right)$ is a directed path between players $i$ and $j, i \neq j$, in a permission structure $D$ if $i_{1}=i, i_{k}=j$ and $\left(i_{h}, i_{h+1}\right) \in D$ for all $1 \leq h \leq k-1$. A permission structure $D$ is acyclic if there exists no directed path $\left(i_{1}, \ldots, i_{k}\right)$ with $\left(i_{k}, i_{1}\right) \in D$. Note that in an acyclic permission structure there can be more than one directed path from player $i$ to player $j \neq i$. Also note that in an acyclic permission structure $D$ there always exists at least one player with no predecessors, i.e. $T O P(D):=\left\{i \in N \mid P_{D}(i)=\emptyset\right\} \neq \emptyset$. We refer to these players as the top-players in the permission structure.

Two approaches to games with a permission structure have been considered. In the conjunctive approach as developed in Gilles, Owen and van den Brink (1992) and van den Brink and Gilles (1996), it is assumed that each player needs permission from all its predecessors in order to cooperate. This implies that a coalition $S \subseteq N$ is feasible if and only if for every player $S$ all its predecessors belong to $S$. The set of feasible coalitions in this approach is therefore given by

$$
\Phi_{D}^{c}:=\left\{S \subseteq N \mid P_{D}(i) \subset S \text { for all } i \in S\right\},
$$

which we refer to as the conjunctive feasible set of $D$.

Alternatively, in the disjunctive approach as developed in Gilles and Owen (1994) and van den Brink (1997), it is assumed that each player (except the top-players) needs permission from at least one of its predecessors before it is allowed to cooperate with other players. Consequently, a coalition is feasible if and only if every player in the coalition (except the top-players) has at least one predecessor who also belongs to the coalition. Thus, the feasible coalitions are the ones in the set

$$
\Phi_{D}^{d}:=\left\{S \subseteq N \mid P_{D}(i) \cap S \neq \emptyset \text { for all } i \in S \backslash T O P(D)\right\},
$$

which we refer to as the disjunctive feasible set of $D$.

Example 2.2 Consider the permission structure $D$ on $N=\{1,2,3,4\}$ given by $D=$ $\{(1,2),(1,3),(2,4),(3,4)\}$, see Figure 2. Then $\Phi_{D}^{c}=\{\{1\},\{1,2\},\{1,3\},\{1,2,3\},\{1,2,3,4\}\}$ and $\Phi_{D}^{d}=\Phi_{D}^{c} \cup\{\{1,2,4\},\{1,3,4\}\}$.

An approach using restricted games similar to the approach of Myerson (1977) for communication graph games assigns to every coalition in a game with a permission structure 


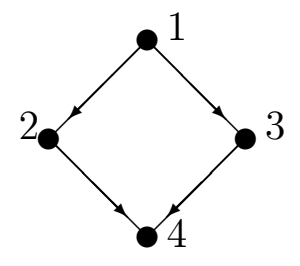

Figure 2: Permission structure $D$ of Example 2.2

the worth of its largest feasible subset. ${ }^{2}$

Algaba, Bilbao, van den Brink and Jiménez-Losada (2004) show that the conjunctive and disjunctive feasible sets in acyclic permission structures are antimatroids. Antimatroids were introduced by Dilworth (1940) as particular examples of semimodular lattices. Since then, several authors have obtained the same concept by abstracting various combinatorial situations (see Korte, Lovász, and Schrader (1991) and Edelman and Jamison (1985)).

Definition 2.3 $A$ set system $\mathcal{A} \subseteq 2^{N}$ is an antimatroid if it satisfies the following properties

(feasible empty set) $\emptyset \in \mathcal{A}$,

(union closedness) $S, T \in \mathcal{A}$ implies that $S \cup T \in \mathcal{A}$,

(accessibility) $S \in \mathcal{A}$ with $S \neq \emptyset$, implies that there exists $i \in S$ such that $S \backslash\{i\} \in \mathcal{A}$.

Union closedness means that the union of two feasible coalitions is also feasible. Accessibility means that every nonempty feasible coalition has at least one player that can leave such that the set of remaining players is a feasible subcoalition. A player that can leave a coalition $S$ leaving behind another feasible coalition is called an extreme player.

Definition 2.4 A player $i \in S$, with $S \in \mathcal{F}, \mathcal{F} \subseteq 2^{N}$, such that $S \backslash\{i\} \in \mathcal{F}$ is called an extreme player of coalition $S$ in $\mathcal{F}$.

The set of extreme players of $S \in \mathcal{F}$ is denoted by $\operatorname{ex}(S)=\{i \in S \mid S \backslash\{i\} \in \mathcal{F}\}$. By accessibility every nonempty feasible coalition in an antimatroid has at least one extreme player. A coalition that has exactly one extreme player is called a path.

\footnotetext{
${ }^{2}$ Whereas in communication graphs every coalition can be partitioned into feasible components, in a conjunctive- or disjunctive permission structure every coalition has a unique largest feasible subset.
} 
Definition 2.5 A coalition $S \in \mathcal{F}, \mathcal{F} \subseteq 2^{N}$ is a path if $\mid$ ex $(S) \mid=1$. The path $S \in \mathcal{F}$ is called an $i$-path if it has $i \in S$ as unique extreme player.

Let $(N, \mathcal{A})$ be an antimatroid and let $S, T \in \mathcal{A}$ be such that $|S|<|T|$. Accessibility implies an ordering $T=\left\{i_{1}, \ldots, i_{t}\right\}$ with $\left\{i_{1}, \ldots, i_{j}\right\} \in \mathcal{A}$ for $j=1, \ldots, t$. Let $k \in$ $\{1, \ldots, t\}$ be the minimum index with $i_{k} \notin S$. Then $S \cup\left\{i_{k}\right\}=S \cup\left\{i_{1}, \ldots, i_{k}\right\} \in \mathcal{A}$ by union closedness. Therefore, the definition of antimatroid implies that for every $S, T \in \mathcal{A}$ with $|S|<|T|$ there exists $i \in T \backslash S$ such that $S \cup\{i\} \in \mathcal{A}$. In particular, for $S, T \in \mathcal{A}$ such that $|T|=|S|+1$ there is an element $j \in T \backslash S$ such that $S \cup\{j\} \in \mathcal{A}$.

Finally, a set system $(N, \mathcal{F})$ is called normal if $N=\bigcup_{S \in \mathcal{F}} S$, i.e. if every player belongs to at least one feasible coalition. ${ }^{3}$ Notice that if $(N, \mathcal{A})$ is a normal antimatroid then union closedness implies that $N \in \mathcal{A}$.

\subsection{Comparing communication and hierarchies}

Let $\mathcal{F} \subseteq 2^{N}$ be an arbitrary set of feasible coalitions. Since all singletons in a communication graph are connected, it follows that communication feasible sets arising from communication graphs contain the empty set and satisfy normality, i.e. every player belongs to at least one feasible coalition. Further, communication feasible sets also satisfy accessibility. They even satisfy the stronger 2-accessibility meaning that every feasible coalition with two or more players has at least two players that can leave the coalition such that the set of remaining players is a feasible coalition. Communication feasible sets are not union closed (as is illustrated by the two connected coalitions $\{1,2\}$ and $\{5\}$ in Example 2.1 whose union is not connected). However, as shown by Algaba, Bilbao, Borm and López (2001), communication feasible sets satisfy the weaker union stability meaning that the union of two feasible coalitions that have a nonempty intersection is also feasible. In van den Brink (2012) it is shown that a set system is a communication feasible set if and only if it contains the empty set and satisfies normality, union stability and 2-accessibility.

Theorem 2.6 [van den Brink (2012)] Let $\mathcal{F} \subseteq 2^{N}$ be a normal set of feasible coalitions on $N \subset \mathbb{N}$. Then $\mathcal{F}$ is the communication feasible set of some communication graph if and only if it satisfies the following properties:

(feasible empty set) $\emptyset \in \mathcal{F}$,

(union stability) $S, T \in \mathcal{F}$ with $S \cap T \neq \emptyset$ implies that $S \cup T \in \mathcal{F}$,

\footnotetext{
${ }^{3}$ For results of games on antimatroids we refer to Algaba, Bilbao, van den Brink and Jiménez-Losada $(2003,2004)$. For antimatroids that are not normal similar results can be stated restricted to the class of players that belong to at least one feasible coalition.
} 
(2-accessibility) $S \in \mathcal{F}$ with $|S| \geq 2$ implies that there exist $i, j \in S, i \neq j$, such that $S \backslash\{i\} \in \mathcal{F}$ and $S \backslash\{j\} \in \mathcal{F}$.

Comparing Theorem 2.6 with Definition 2.3, we conclude that communication feasible sets are characterized by properties similar to the ones used in defining antimatroids. Besides normality and feasibility of the empty set, both satisfy an accessibility and a union property. Obviously, 2-accessibility implies accessibility and therefore communication feasible sets satisfy a stronger accessibility property. However, antimatroids satisfy union closedness instead of union stability, and therefore antimatroids satisfy a stronger union property.

Usually the set of links, being coalitions of size two, is considered as the basis of a communication network. Note that by applying 2-accessibility, we can generate these bilateral links from any communication feasible set.

Also note that given 2-accessibility, normality implies that $\{i\} \in \mathcal{F}$ for all $i \in N$ as is the case for communication feasible sets. Given union closedness, normality implies that $N \in \mathcal{F}$ as is the case for antimatroids.

\section{Accessible union stable systems}

The goal of this paper is to get a better understanding of organizations that have hierarchical as well as communication features. Therefore, we study those set systems satisfying the weaker union and accessibility properties discussed in the previous section. Note that both are satisfied by all communication feasible sets and antimatroids. We formally introduce accessible union stable systems as those set systems that contain the empty set and satisfy union stability and accessibility. ${ }^{4}$

Definition 3.1 $A$ set system $\mathcal{F} \subseteq 2^{N}$ is an accessible union stable system if it satisfies the following properties:

(feasible empty set) $\emptyset \in \mathcal{F}$,

(union stability) $S, T \in \mathcal{F}$ with $S \cap T \neq \emptyset$, implies that $S \cup T \in \mathcal{F}$,

(accessibility) $S \in \mathcal{F}$ with $S \neq \emptyset$, implies that there exists $i \in S$ such that $S \backslash\{i\} \in \mathcal{F}$.

Obviously, antimatroids and communication feasible sets are accessible union stable systems. Other examples of accessible union stable systems on $N=\{1, \ldots, n\}$ are $\mathcal{F}=2^{N}$, $\mathcal{F}=\{\emptyset,\{i\}\}$ for $i \in N$, and $\mathcal{F}=\{\emptyset,\{1\}, \ldots,\{n\}\}$.

\footnotetext{
${ }^{4}$ For easy reading we repeat in every definition the set properties such as union stability, accessibility etc., when they are used.
} 
Union stability has a natural and intuitive interpretation in the context of partial cooperation since common players will be vital to establish communication in the union coalition. But only requiring union stability it is not clear what is the hierarchical feature of an organization. In the new structures, the hierarchy is reflected by accessibility. By definition the following holds.

Proposition 3.2 (i) An accessible union stable system is an antimatroid if and only if it is union closed. (ii) A normal accessible union stable system is a communication feasible set if and only if it satisfies 2-accessibility.

Since accessible union stable systems generalize communication feasible sets as well as antimatroids, they can help us to study organizations that have hierarchical as well as communication features. Next, we will show how accessible union stable systems are related to other structures in the literature. An already existing class that contains communication feasible sets and antimatroids are the augmenting systems introduced by Bilbao (2003).

Definition 3.3 A set system $\mathcal{F} \subseteq 2^{N}$ is an augmenting system if it satisfies the following properties:

(feasible empty set) $\emptyset \in \mathcal{F}$,

(union stability) $S, T \in \mathcal{F}$ with $S \cap T \neq \emptyset$, implies that $S \cup T \in \mathcal{F}$,

(augmentation 1) $S, T \in \mathcal{F}$ with $S \subset T$, implies that there exists $i \in T \backslash S$ such that $S \cup\{i\} \in \mathcal{F}$.

Augmentation 1 establishes that whenever there are two feasible coalitions such that one is contained in the other, we can keep adding players from the 'bigger' coalition to the 'smaller' coalition one by one, such that after each addition the new coalition is feasible. This property can be used in defining solutions for games that are based on marginal vectors (such as the Shapley value). Assuming that the 'grand coalition' $N$ is feasible one can, starting with the empty set and adding one player at each step, define a sequence of feasible coalitions ending up in the 'grand coalition'. This means that we can always define a permutation $\pi: N \longrightarrow N$ such that $\{\pi(1), \ldots, \pi(k)\}$ is a feasible coalition for every $k \in\{1, \ldots, n\}$. We will see later that the same cannot be done for arbitrary accessible union stable systems.

Players who can be joined to a feasible coalition keeping feasibility are called augmentation points. 
Definition 3.4 Let $\mathcal{F} \subseteq 2^{N}$ be an accessible union stable system. A player $i \in N \backslash S$, with $S \in \mathcal{F}$, such that $S \cup\{i\} \in \mathcal{F}$ is called an augmentation point of coalition $S$ in $\mathcal{F}$.

Given $S \in \mathcal{F}$, we denote by $a u(S)=\{i \in N \backslash S \mid S \cup\{i\} \in \mathcal{F}\}$ the set of augmentation points of $S$. Note that augmentation 1 implies accessibility. Indeed, for $T \in \mathcal{F}$ with $T \neq \emptyset$, by the augmentation 1 property, we have that there exists a sequence of coalitions $T_{0}, T_{1}, \ldots, T_{t}$, with $T_{h} \in \mathcal{F},\left|T_{h}\right|=h, 0 \leq h \leq t$, such that $\emptyset=T_{0} \subset T_{1} \subset \cdots \subset T_{t-1} \subset$ $T_{t}=T$. Therefore, there exists a player $i \in T$ such that $T \backslash\{i\}=T_{t-1} \in \mathcal{F}$. This shows that augmenting systems satisfy accessibility, and therefore they are accessible union stable systems.

Proposition 3.5 If $\mathcal{F} \subseteq 2^{N}$ is an augmenting system then $\mathcal{F}$ is an accessible union stable system.

However, accessibility does not imply augmentation 1 , and therefore the reverse is not true, i.e. there are accessible union stable systems that are not augmenting systems. The next example discusses an application of accessible union stable systems that are neither an augmenting system nor an antimatroid nor a communication feasible set.

Example 3.6 (Exploring and careful societies) Consider two societies of players, say $N$ and $M, N \cap M=\emptyset$. Every subset of each society can form a feasible coalition. Further, every subset of society $N$ can form a coalition 'outside' $N$, but the players in $M$ can only form a feasible coalition with 'outside' players when they join together. Therefore, we can consider society $N$ as 'explorers' who each can go individually or with any group to the 'outside world', and society $M$ as a 'careful' society who can only go out together.

The corresponding set of feasible coalitions is an accessible union stable system. However, (i) it is not the set of connected coalitions of a communication graph (since it does not satisfy 2-accessibility), (ii) it is not an antimatroid (since it is not union closed), and (iii) it is not an augmenting system (since it does not satisfy augmentation 1).

This is illustrated by the following example with two societies. Let $N=\{1,2\}$ be a society of explorers, and $M=\{3,4,5\}$ a society of careful players. Assuming that all coalitions within each society are feasible, but a coalition containing players from both societies can only be formed if it contains all players of $M$ (and any subset of $N$ ), the resulting set of feasible coalitions is

$$
\begin{aligned}
\mathcal{F}= & \{\emptyset,\{1\},\{2\},\{1,2\},\{3\},\{4\},\{5\},\{3,4\},\{3,5\},\{4,5\},\{3,4,5\}, \\
& \{1,3,4,5\},\{2,3,4,5\},\{1,2,3,4,5\}\}
\end{aligned}
$$


(where the first row of $\mathcal{F}$ contains $N, M$ and all their subsets, while in the second row are the coalitions that contain players from both societies.)

This set system is union stable since (i) any union of two coalitions that are either a subset of $N$ or a subset of $M$ are feasible, and (ii) the union of two nondisjoint feasible coalitions containing players from $N$ and $M$ must contain all players from $M$ (since one of these two coalitions must contain players from $N$ and $M$, it must contain all players from $M$ ), and therefore is feasible.

The set system is accessible since (i) for each non-empty feasible coalition that is a subset of $N$ or $M$ every player in this coalition is an extreme player (i.e., it can be deleted keeping feasibility), and (ii) for every feasible coalition that contains players from both $N$ and $M$ each player from $N$ can be deleted. The resulting coalition still contains all players from $M$, and therefore is feasible.

To show that the set system $\mathcal{F}$ is not a communication feasible set, consider for example feasible coalition $\{2,3,4,5\}$. This coalition contains one player from $N$, player 2 , and all players from $M$. But since none of the players of $M$ can be deleted, player 2 is the only extreme player, so the set system does not satisfy 2-accessibility.

To show that the set system $\mathcal{F}$ is not an antimatroid, consider for example coalitions $\{1\}$ and $\{3\}$. These are proper subsets of $N$, respectively $M$, so their union contains a player from $N$ and a player from $M$ but does not contain all players from $M$, and therefore is not feasible showing that $\mathcal{F}$ does not satisfy union closedness.

Finally, to show that the set system $\mathcal{F}$ is not an augmenting system, consider coalitions $N=\{1,2\}$ and $N \cup M=\{1,2,3,4,5\}$. Then no single player from $M$ can be added to $N$ to get a feasible coalition since the players of $M$ only join $N$ as group, showing that it does not satisfy augmentation 1.

In the next example, we illustrate the difference in coalition formation between accessible union stable systems and augmenting systems. In fact, accessible union stable systems provide flexibility to players who belong to the set of 'starting players' as well as the possibility of choosing partners outside the set of starting players whereas in augmenting systems the set of starting players can not be chosen freely in all cases nor can the partners outside the set of starting players be incorporated one by one in the coalitional formation process.

Example 3.7 Consider the set systems $\mathcal{F}_{1}, \mathcal{F}_{2}$ on $N=\{1,2,3,4\}$ given by

$$
\begin{aligned}
& \mathcal{F}_{1}=\{\emptyset,\{2\},\{4\},\{1,2\},\{3,4\},\{1,2,3\},\{2,3,4\}, N\} . \\
& \mathcal{F}_{2}=\{\emptyset,\{1\},\{4\},\{1,2\},\{3,4\},\{1,2,3\},\{2,3,4\}, N\} .
\end{aligned}
$$


Both of them are accessible union stable systems but whereas $\mathcal{F}_{1}$ is not an augmenting system the set system $\mathcal{F}_{2}$ is an augmenting system. Observe that the only difference between $\mathcal{F}_{1}$ and $\mathcal{F}_{2}$ is the set of 'starting players' in the coalitional formation process.

Another interesting relation between accessible union stable systems and antimatroids is that every set system such that the collection of feasible coalitions in this system containing a particular player is an antimatroid, is an accessible union stable system.

Proposition 3.8 Let $\mathcal{F} \subseteq 2^{N}$ be a set system containing the empty set. If $\mathcal{F}_{i}=\{\emptyset\} \cup\{T \in$ $\mathcal{F} \mid i \in T\}$ is an antimatroid for all $i \in N$, then $\mathcal{F}$ is an accessible union stable system.

Proof: Consider $S, T \in \mathcal{F}$ with $S \cap T \neq \emptyset$. If $j \in S \cap T$ then $S, T \in \mathcal{F}_{j}$. Since $\mathcal{F}_{j}$ is union closed it holds that $S \cup T \in \mathcal{F}_{j}$ and hence $S \cup T \in \mathcal{F}$, showing union stability of $\mathcal{F}$.

To show accessibility of $\mathcal{F}$, consider $S \in \mathcal{F}, S \neq \emptyset$. Then $S \in \mathcal{F}_{j}$ for some $j \in S$. But then there exists an $h \in S$ such that $S \backslash\{h\} \in \mathcal{F}_{j}$ by accessibility of $\mathcal{F}_{j}$. Hence, $S \backslash\{h\} \in \mathcal{F}$, showing accessibility of $\mathcal{F}$.

The reverse is not true as shown in the following example.

Example 3.9 Consider set system $\mathcal{F}$ with $N=\{1,2,3\}$ given by $\mathcal{F}=\{\emptyset,\{1\},\{2\},\{2,3\}, N\}$. This is an accessible union stable system but $\mathcal{F}_{1}=\{\{1\}, N\}$ is not an antimatroid since it fails accessibility ( $n o$ single player can be deleted from $N$ ).

The dual structure of a set system $\mathcal{F} \subseteq 2^{N}$ is the set system $\mathcal{F}^{d}$ given by

$$
\mathcal{F}^{d}=\{S \subseteq N \mid N \backslash S \in \mathcal{F}\}
$$

It is known that convex geometries are the dual structures of antimatroids. Convex geometries are a combinatorial abstraction of convex sets introduced by Edelman and Jamison (1985).

Definition 3.10 A set system $\mathcal{G} \subseteq 2^{N}$ is a convex geometry if it satisfies the following properties:

(feasible empty set) $\emptyset \in \mathcal{G}$,

(intersection closed) $S, T \in \mathcal{G}$ implies that $S \cap T \in \mathcal{G}$,

(augmentation 2) $S \in \mathcal{G}$ with $S \neq N$, implies that there exists $i \in N \backslash S$ such that $S \cup\{i\} \in \mathcal{G}$. 
So, a convex geometry is a set system that contains the empty set, is intersection closed and satisfies an alternative augmentation property saying that for every feasible coalition that is a proper subset of the 'grand coalition' $N$ there is a player that can be added resulting in a feasible coalition of higher cardinality. ${ }^{5}$ Note that this implies that the 'grand coalition' is feasible, and thus convex geometries are normal set systems. Also, note that an augmenting system that is intersection closed is a convex geometry.

If an accessible union stable system $(N, \mathcal{F})$ is a convex geometry then $\mathcal{F}$ is closed under intersection and $N \in \mathcal{F}$. However, the reverse it is not true as the following example illustrates.

Example 3.11 Let $N=\{1,2,3\}$ and $\mathcal{F}=\{\emptyset,\{1\},\{2\},\{3\},\{1,2\}, N\}$. This is an accessible union stable system that is closed under intersection with $N \in \mathcal{F}$. But au $(\{3\})=$ $\emptyset$, and therefore it does not satisfy augmentation 2 and it is not a convex geometry.

It turns out that the dual structures of accessible union stable systems form a class of set systems that contain all convex geometries.

Definition 3.12 $A$ set system $\mathcal{F} \subseteq 2^{N}$ is an intersecting stable system with the augmentation property if it satisfies the following properties:

(feasible empty set) $\emptyset \in \mathcal{F}$,

(weak intersection closed) $S, T \in \mathcal{F}$ with $S \cup T \neq N$, implies that $S \cap T \in \mathcal{F}$

(augmentation 2) $S \in \mathcal{F}$ with $S \neq N$, implies that there exists $i \in N \backslash S$ such that $S \cup\{i\} \in \mathcal{F}$.

Proposition 3.13 Let $\mathcal{F} \subseteq 2^{N}$ be a set system with $N \in \mathcal{F}$. Then $\mathcal{F}$ is an accessible union stable system if and only if $\mathcal{F}^{d}$ is an intersecting stable system with the augmentation property.

Proof: The sets $\emptyset$ and $N$ belong to $\mathcal{F}$, and thus also to $\mathcal{F}^{d}$. Note that

(i) $S, T \in \mathcal{F} \Leftrightarrow(N \backslash S),(N \backslash T) \in \mathcal{F}^{d}$,

(ii) $S \cap T \neq \emptyset \Leftrightarrow(N \backslash S) \cup(N \backslash T) \neq N$, and

(iii) $S \cup T \in \mathcal{F} \Leftrightarrow N \backslash(S \cup T)=(N \backslash S) \cap(N \backslash T) \in \mathcal{F}^{d}$.

\footnotetext{
${ }^{5}$ Edelman and Jamison (1985) showed that $L$ is a connected block graph if and only if the collection of subsets of $N$ which induces connected subgraphs is a convex geometry.
} 
Therefore, union stability of $\mathcal{F}$ implies that $\mathcal{F}^{d}$ is weak intersection closed.

Finally, since for any $S \subseteq N$ with $i \in S, N \backslash S \neq N, i \in N \backslash(N \backslash S)=S$ and,

(iv) $S, S \backslash\{i\} \in \mathcal{F} \Leftrightarrow N \backslash S \in \mathcal{F}^{d}, N \backslash(S \backslash\{i\})=(N \backslash S) \cup\{i\} \in \mathcal{F}^{d}$,

accessibility of $\mathcal{F}$ implies that $\mathcal{F}^{d}$ satisfies augmentation 2.

We refer to set systems that satisfy the properties of the above theorem (i.e. whose dual structure is an accessible union stable system) as an intersecting stable system with the augmentation property. Note that every convex geometry is such an intersecting stable system with the augmentation property since it satisfies a stronger intersection closedness. However, not every intersecting stable system with the augmentation property is a convex geometry as shown by the next example.

Example 3.14 Consider the digraph $(N, D)$ with $N=\{1,2,3\}$ and $D=\{(1,3),(2,3)\}$. Suppose that the feasible coalitions are those that are connected ${ }^{6}$ and disjunctive feasible, i.e. whenever player 3 is in a coalition either player 1 or player 2 (or both) should be in the coalition. (So, they are the disjunctive feasible coalitions except $\{1,2\}$.) The resulting set system is

$$
\mathcal{F}^{d}=\{\emptyset,\{1\},\{2\},\{1,3\},\{2,3\},\{1,2,3\}\} .
$$

This set system is weak intersection closed and satisfies augmentation 2. However, since the intersection of the two feasible coalition $\{1,3\}$ and $\{2,3\}$ (being singleton $\{3\}$ ) is not feasible, this set system is not intersection closed and thus not a convex geometry.

Using the definition of convex geometries and intersecting stable systems with the augmentation property, the following proposition follows straightforwardly.

Proposition 3.15 An intersecting stable system with the augmentation property $\mathcal{F} \subseteq 2^{N}$ is a convex geometry if and only if $\mathcal{F}$ is closed under intersection.

Given an intersecting stable system with the augmentation property $\mathcal{F} \subseteq 2^{N}$, by augmentation 2 the sets $a u(S) \neq \emptyset$, for all $S \in \mathcal{F}, S \neq N$. However, the set of extreme points of some coalitions can be empty.

\footnotetext{
${ }^{6}$ A coalition $S$ is connected in digraph $D$ if for every $i, j \in S, i \neq j$, there is a sequence of players $\left(i_{1}, \ldots, i_{k}\right)$ such that $\left\{i_{1}, \ldots, i_{k}\right\} \subseteq S, i_{1}=i, i_{k}=j$, and $\left\{\left(i_{h}, i_{h+1}\right),\left(i_{h+1}, i_{h}\right)\right\} \cap D \neq \emptyset$ for $h=$ $1, \ldots, k-1$.
} 
Example 3.16 Consider the set system $\mathcal{F}^{d}=\{\emptyset,\{1\},\{1,3\},\{2,3\}, N\}$ on $N=\{1,2,3\}$. This is an intersecting stable system with the augmentation point property and ex $(\{2,3\})=$ $\emptyset$.

Notice that if an intersecting stable system with the augmentation property is an antimatroid then it is closed under union. The reverse it is not true as the following example shows.

Example 3.17 Consider the set system $\mathcal{F}=\{\emptyset,\{3\},\{1,2\},\{1,3\},\{2,3\}, N\}$ on $N=$ $\{1,2,3\}$. This set system is closed under union, and it is an intersecting stable system with the augmentation property but, since ex $(\{1,2\})=\emptyset$, it does not satisfy accessibility and therefore it is not an antimatroid.

\section{The supports of an accessible union stable system}

Let $(N, \mathcal{F})$ be an accessible union stable system and $\mathcal{G} \subseteq \mathcal{F}$. Since $\mathcal{F}$ is union stable, we can inductively define the following families $\mathcal{G}^{(m)}, m=0,1, \ldots$, in the same way as Algaba et al. (2000) did for arbitrary union stable systems, i.e.,

$$
\mathcal{G}^{(0)}=\mathcal{G}, \quad \mathcal{G}^{(m)}=\left\{S \cup T \mid S, T \in \mathcal{G}^{(m-1)}, S \cap T \neq \emptyset\right\}, \quad(m=1,2, \ldots)
$$

Notice that $\mathcal{G}^{(0)} \subseteq \mathcal{G}^{(m-1)} \subseteq \mathcal{G}^{(m)} \subseteq \mathcal{F}$, since $\mathcal{G} \subseteq \mathcal{F}$ and $\mathcal{F}$ is union stable. The inductive process is finite because $\mathcal{F}$ is finite.

Let $(N, \mathcal{F})$ be an accessible union stable system and let $\mathcal{G} \subseteq \mathcal{F}$. The closure $\overline{\mathcal{G}}$ is given by $\overline{\mathcal{G}}=\mathcal{G}^{(k)}$, where $k$ is defined as the smallest integer such that $\mathcal{G}^{(k+1)}=\mathcal{G}^{(k)}$.

Example 4.1 Consider the accessible union stable system $\mathcal{F}$ on $N=\{1,2,3,4,5\}$ given by:

$$
\mathcal{F}=\left\{\begin{array}{c}
\emptyset,\{1\},\{2\},\{4\},\{5\},\{1,2\},\{3,4\},\{4,5\},\{2,3,4\}, \\
\{3,4,5\},\{1,2,3,4\},\{2,3,4,5\}, N
\end{array}\right\} .
$$

For the family $\mathcal{G}=\{\emptyset,\{1\},\{4\},\{1,2\},\{3,4,5\},\{1,2,3,4\}\}$, note that

$$
\begin{aligned}
& \mathcal{G}^{(0)}=\mathcal{G}, \\
& \mathcal{G}^{(1)}=\mathcal{G} \cup\{\{1,2,3,4,5\}\}, \\
& \mathcal{G}^{(2)}=\mathcal{G}^{(1)} \text { and } \mathcal{G}^{(1)}=\overline{\mathcal{G}}=\mathcal{F} .
\end{aligned}
$$

Let $(N, \mathcal{F})$ be an accessible union stable system. Then there can be feasible coalitions which can be written as the union of two feasible coalitions with a nonempty intersection. We consider the set

$$
R(\mathcal{F})=\{R \in \mathcal{F} \mid R=S \cup T, S \neq R, T \neq R, S, T \in \mathcal{F}, S \cap T \neq \emptyset\}
$$


consisting of those feasible coalitions which can be written as the union of two distinct feasible coalitions with a nonempty intersection. The basis of an accessible union stable system consists of those coalitions that cannot be written as the union of two feasible coalitions with a nonempty intersection. ${ }^{7}$

Definition 4.2 Let $\mathcal{F} \subseteq 2^{N}$ be an accessible union stable system. The elements of the set $B(\mathcal{F})=\mathcal{F} \backslash R(\mathcal{F})$ are called the supports of $\mathcal{F}$.

By construction, the set $B(\mathcal{F})$ is unique, nonempty if $\mathcal{F}$ is nonempty, and satisfies the following properties:

1. If $\emptyset \in \mathcal{F}$, then $\emptyset \in B(\mathcal{F})$.

2. If $S \in \mathcal{F}$ is a minimal element in $(\mathcal{F}, \subseteq)$, then $S \in B(\mathcal{F})$.

3. If $S \in \mathcal{F}$ and $|S| \leq 2$, then $S \in B(\mathcal{F})$.

Next, the following characterization of the set of supports can be obtained in the same way as for union stable systems (see Algaba, Bilbao, Borm and López (2001)) and follows directly from the definition of $B(\mathcal{F})$.

Proposition 4.3 Let $\mathcal{F} \subseteq 2^{N}$ be an accessible union stable system and $B(\mathcal{F})$ the set of its supports. Then $B(\mathcal{F})$ is the minimal subset of $\mathcal{F}$ such that $\overline{B(\mathcal{F})}=\mathcal{F}$.

Example 4.4 Consider the accessible union stable system $\mathcal{F}$ on $N=\{1,2,3,4,5\}$ given by

$$
\mathcal{F}=\left\{\begin{array}{c}
\emptyset,\{1\},\{2\},\{4\},\{5\},\{1,2\},\{3,4\},\{4,5\},\{2,3,4\}, \\
\{3,4,5\},\{1,2,3,4\},\{2,3,4,5\}, N
\end{array}\right\} .
$$

The supports of $\mathcal{F}$ are given by

$$
B(\mathcal{F})=\{\emptyset,\{1\},\{2\},\{4\},\{5\},\{1,2\},\{3,4\},\{4,5\},\{2,3,4\}\} .
$$

We introduced accessible union stable systems as a model that generalizes communication feasible sets as well as antimatroids in such a way that two defining properties reflect communication (union stability) and hierarchy (accessibility). Next, we want to see how these features influence the basis of the system. It is known that the supports of a communication feasible set are exactly those elements that have cardinality one or two, the first type being the singletons and the second type being the links or edges of the communication graph. The supports of an antimatroid are the paths being those feasible coalitions that have exactly one extreme player.

\footnotetext{
${ }^{7}$ In fact, such a basis can be defined for every union stable system.
} 
Example 4.5 Consider the permission structure of Example 2.2. The paths in the conjunctive feasible set are $\{1\},\{1,2\},\{1,3\}$ and $\{1,2,3,4\}$, and every player is extreme in one path. ${ }^{8}$ The paths in the disjunctive feasible set are $\{1\},\{1,2\},\{1,3\},\{1,2,4\}$ and $\{1,3,4\}$. Note that these paths coincide with the directed paths in a permission structure as defined in the preliminaries.

Applying accessibility, it holds that $\operatorname{ex}(S) \neq \emptyset$, for any nonempty $S \in \mathcal{F}$, where $\operatorname{ex}(S)$ is the set of extreme players of $S$ in $\mathcal{F}$. For accessible union stable systems it turns out that every support either has cardinality at most two or is a path. (In the accessible union stable system of Example 4.4, the supports with cardinality at most two are those in $B(\mathcal{F}) \backslash\{\{2,3,4\}\}$, while support $\{2,3,4\}$ is a path.)

Proposition 4.6 Let $\mathcal{F} \subseteq 2^{N}$ be an accessible union stable system. If $B \in B(\mathcal{F})$ with $|B|>2$ then $|\operatorname{ex}(B)|=1$, i.e., $B$ is a path.

Proof: Suppose that $B \in B(\mathcal{F})$ is a support of $\mathcal{F}$ such that $|B|>2$ and $|e x(B)|>1$, i.e., $B$ has at least two extreme points. Then there exists $i, j \in B$, with $i \neq j$, such that $B \backslash\{i\} \in \mathcal{F}$ and $B \backslash\{j\} \in \mathcal{F}$. Therefore

$$
(B \backslash\{i\}) \cup(B \backslash\{j\})=B \text { and }(B \backslash\{i\}) \cap(B \backslash\{j\})=B \backslash\{i, j\} \neq \emptyset,
$$

which contradicts the fact that $B$ is a support of $\mathcal{F}$.

The reverse is not true, i.e., not every path with more than two players is a support.

Example 4.7 Consider the set $N=\{1,2,3,4\}$ and the accessible union stable system given by $\mathcal{F}=\{\{1\},\{2\},\{4\},\{1,2\},\{3,4\},\{2,3,4\}, N\}$.

Its basis is $B(\mathcal{F})=\{\{1\},\{2\},\{4\},\{1,2\},\{3,4\},\{2,3,4\}\}$. Since the only extreme player of the 'grand coalition' $N$ is player 1, the grand coalition is a path but it is not a support.

\section{Cycle-free accessible union stable systems}

Algaba, Bilbao, Borm and López (2001) considered the subclass of union stable systems that are intersection closed (if the intersection contains at least two elements) and such that every non-unitary feasible coalition can be written in a unique way as a union of non-unitary supports. A coalition $S$ is non-unitary if $|S| \neq 1$ (i.e. $S$ is not a singleton). Note that for set systems $\mathcal{F}$ in this class, if $S, T \in B(\mathcal{F}),|S| \geq 2$, then $S \not \subset T$ (since otherwise $T$ could be written as $T \cup \emptyset$ as well as $T \cup S$, and then the representation would not be unique.)

\footnotetext{
${ }^{8}$ In fact, the poset antimatroids are characterized as those antimatroids such that every player has exactly one path, see Algaba, Bilbao, van den Brink and Jiménez-Losada (2004).
} 
Definition 5.1 A union stable system $\mathcal{F} \subseteq 2^{N}$ belongs to the subclass of union stable systems $U S I^{N}$ if the following two conditions are satisfied:

(2-intersection closed) $S, T \in \mathcal{F}$ with $|S \cap T| \geq 2$ implies that $S \cap T \in \mathcal{F}$,

(cycle-free) every non-unitary feasible coalition can be written in a unique way as a union of non-unitary supports.

Algaba, Bilbao, Borm and López (2001) introduce this class as a generalization of cycle-free undirected graphs in the sense that the set of connected coalitions in any cycle-free graph satisfies these properties. This subclass of accessible union stable cooperation systems contains the important class of connected coalitions in a cycle-free communication graph, see e.g. Le Breton, Owen and Weber (1992) and Demange $(1994,2004),{ }^{9}$ respectively a tree.

Next we motivate why we call the second property cycle-freeness of a set system. In van den Brink (2012) it is shown that adding intersection closedness to the properties mentioned in Theorem 2.6 characterizes the sets of coalitions that can be the set of connected coalitions in a cycle-complete graph. A graph is cycle-complete if, whenever there is a cycle, the subgraph on that cycle is complete. ${ }^{10}$

Proposition 5.2 [van den Brink (2012)] Let $\mathcal{F} \subseteq 2^{N}$ be a set of feasible coalitions. Then $\mathcal{F}$ satisfies normality, 2-accessibility, union stability and intersection closedness if and only if there is a cycle-complete graph $L$ such that $\mathcal{F}=\mathcal{F}_{L}$.

Note that every cycle-free communication graph is cycle-complete. Cycle-complete structures are often encountered in the economic literature, for example, cycle-free structures in, e.g. auction situations (see Graham, Marshall and Richard (1990)), airport games (see Littlechild and Owen (1973)), sequencing games (see Curiel, Potters, Rajendra Prasad, Tijs and Veltman $(1993,1994)$ ), water distribution problems (see Ambec and Sprumont (2002))

\footnotetext{
${ }^{9}$ Le Breton, Owen and Weber (1992) and Demange (1994, 2004), consider a restricted Core concept where coalitional stability is required only for feasible (i.e. connected) coalitions, i.e. they consider the solution $C(N, v, L)=\left\{x \in \mathbb{R}^{N} \mid \sum_{i \in T} x_{i}=v(T)\right.$ for all $T \in C_{L}(N)$, and $\sum_{i \in S} x_{i} \geq v(S)$ for all $\left.S \in \mathcal{F}_{L}\right\}$. They show that this set of Core payoff vectors is nonempty if the game is superadditive and the communication graph is cycle-free. Under these conditions this solution coincides with the (unrestricted) Core of the restricted game $\left(N, v_{L}\right)$, see also Kaneko and Wooders (1982). Demange (2004) also shows that in case the communication graph contains a cycle, one can always find a superadditive game such that the corresponding set of Core payoff vectors is empty. This is interesting since, as mentioned before, superadditivity of a game $v$ does not guarantee the existence of a Core-stable payoff vector for $v$.

${ }^{10}$ In Algaba, Bilbao, van den Brink and Jiménez-Losada (2004) it is shown that the class of antimatroids that are intersection closed are exactly the poset antimatroids, that is, the antimatroids that can be the conjunctive feasible set of some acyclic permission structure.
} 
or polluted river problems (see Ni and Wang (2007)), and other cycle-complete structures are encountered in, e.g. favor exchange networks (see Jackson, Rodríguez Barraquer and $\operatorname{Tan}(2012))$.

Since under the properties of Theorem 2.6 all singletons are feasible, Proposition 5.2 also holds if we use the weaker 2-intersection closedness instead of intersection closedness. It turns out that adding the second property of Definition 5.1 to those of Theorem 2.6 characterizes the sets of coalitions that can be the set of connected coalitions in a cyclefree graph. Therefore we refer to this property of a union stable system as cycle-freeness.

Proposition 5.3 Let $\mathcal{F} \subseteq 2^{N}$ be a communication feasible set, i.e. there is an undirected graph $L$ such that $\mathcal{F}=\mathcal{F}_{L}$. Then $L$ is cycle-free if and only if every non-unitary feasible coalition in $\mathcal{F}$ can be written in a unique way as a union of non-unitary supports.

Proof: Let $\mathcal{F} \subseteq 2^{N}$ be such that there is an undirected graph $L$ with $\mathcal{F}=\mathcal{F}_{L}$. (Only if) Suppose that $L$ is cycle-free. Then for every connected coalition $S \in \mathcal{F}$ the unique way to write $S$ as union of non-unitary supports is $S=\bigcup_{l \in L(S)} l$. (If) Suppose that $L$ is not cycle-free. Let $\left(i_{1}, i_{2}, \ldots, i_{k}\right), k \geq 3$, be a cycle in $L$. Then $S=\bigcup_{t \in\{1, \ldots, k-1\}}\left\{i_{t}, i_{t+1}\right\}=$ $\left\{i_{k}, i_{1}\right\} \cup\left(\bigcup_{t \in\{2, \ldots, k-1\}}\left\{i_{t}, i_{t+1}\right\}\right)$, and thus $S$ can be written as a union of non-unitary supports in at least two ways.

Since every cycle-free graph is cycle-complete, from Propositions 5.2 and 5.3 it follows that, under the properties of Theorem 2.6, cycle-freeness of $\mathcal{F} \subseteq 2^{N}$ implies intersection closedness of $\mathcal{F}$ but not the other way around. For example, $\mathcal{F}=2^{N}$ is intersection closed, but not cycle-free. So, for accessible union stable systems, 2-intersection closedness is superfluous when we want to consider a generalization of cycle-free undirected graphs as in the class $U S I^{N}$. For arbitrary union stable systems cycle-freeness does not imply 2-intersection closedness (and thus not intersection closedness), as shown in the following example.

Example 5.4 Consider a market with one buyer (player 1), two sellers (players 2 and 3) and one intermediary (player 4). Suppose that a nonempty coalition $S \subseteq 2^{N}, N=$ $\{1,2,3,4\}$, is feasible if and only if it contains the buyer, the intermediary and at least one of the two sellers, i.e. $\mathcal{F}=\{\emptyset,\{1,2,4\},\{1,3,4\},\{1,2,3,4\}\}$ with basis $B(\mathcal{F})=$ $\{\emptyset,\{1,2,4\},\{1,3,4\}\}$. This is a union stable set system that is cycle-free, but not 2intersection closed (since $\{1,2,4\} \cap\{1,3,4\}=\{1,4\}$ which is not feasible).

In Proposition 4.6 we saw that the supports of an accessible union stable system are either of cardinality at most equal to 2 (and thus are singletons or links) or are a path. For arbitrary accessible union stable systems, cycle-freeness implies that every nonempty support contains at most two players, and thus is a link or singleton. 
Proposition 5.5 Let $\mathcal{F} \subseteq 2^{N}$ be an accessible union stable system that is cycle-free (i.e. all non-unitary feasible coalitions can be written in a unique way as a union of non-unitary supports). If $S \in B(\mathcal{F})$ then $|S| \leq 2$.

Proof: Let $\mathcal{F} \subseteq 2^{N}$ be an accessible union stable system that is cycle-free. Suppose there is an $S \in B(\mathcal{F})$ such that $|S|>2$. By accessibility of $\mathcal{F}$ there is a $T \in \mathcal{F}$ such that $T=S \backslash\{i\}$ for some $i \in S$. By cycle-freeness and $T \subset S$, it holds that $T \notin B(\mathcal{F})$. But then, there exist nondisjoint supports $T_{1}, T_{2}, \ldots, T_{k} \in B(\mathcal{F}), k \geq 2$ with $T_{l} \cap T_{p} \neq \emptyset$ for all $l, p \in\{1, \ldots, k\}$, such that $T=\bigcup_{p=1}^{k} T_{p}$. But then, the supports $T_{1}, \ldots, T_{k}$ are subsets of $S$ which contradicts cycle-freeness of $\mathcal{F}$.

Proposition 5.5 implies that a cycle-free accessible union stable system in which all singletons are feasible is the set of connected coalitions in some cycle-free graph, and thus for accessible union stable systems with all singletons feasible, cycle-freeness implies intersection closedness. Without the requirement that all singletons are feasible, the set system still is 2-intersection closed.

Corollary 5.6 (i) Set system $\mathcal{F} \subseteq 2^{N}$ is a cycle-free accessible union stable system such that $\{i\} \in \mathcal{F}$ for all $i \in N$ if and only if there is a cycle-free graph $L$ such that $\mathcal{F}=\mathcal{F}_{L}$.

(ii) If set system $\mathcal{F} \subseteq 2^{N}$ is a cycle-free accessible union stable system such that $\{i\} \in \mathcal{F}$ for all $i \in N$ then $\mathcal{F}$ is intersection closed (and thus 2-intersection closed).

(iii) A set system $\mathcal{F} \subseteq 2^{N}$ is a cycle-free accessible union stable system if and only if $\mathcal{F} \in A U S^{N} \cap U S I^{N}$.

It is interesting to point out that, however, that a cycle-free accessible union stable system does not necessarily satisfy intersection closedness as illustrated by the set system $\mathcal{F}=\{\emptyset,\{1\},\{3\},\{1,2\},\{2,3\},\{1,2,3\}\}$ which is a cycle-free accessible union stable system but is not intersection closed since $\{1,2\} \cap\{2,3\}=\{2\} \notin \mathcal{F}$.

Example 3.6 provides an accessible union stable system with all singletons feasible, but which is not cycle-free (since that $\{1,2,3,4,5\}=\{1,3,4,5\} \cup\{1,2\}=\{2,3,4,5\} \cup$ $\{1,2\})$.

It is known from Algaba, Bilbao and Slikker (2010) that within the class of augmenting systems requiring all singletons to be feasible characterizes the class of augmenting systems that can be the set of connected coalitions in some undirected graph.

Proposition 5.7 [Algaba, Bilbao and Slikker (2010)] Let $\mathcal{F} \subseteq 2^{N}$ be an augmenting system. Then $\{i\} \in \mathcal{F}$ for all $i \in N$ if and only if there is an undirected graph $L$ such that $\mathcal{F}=\mathcal{F}_{L}$ 
From Proposition 5.7 and 3.5 (respectively Theorem 2.6) it follows that we obtain connected coalitions from cycle-free graphs as augmenting systems that are cycle-free and contain all singletons.

Corollary 5.8 (i) Set system $\mathcal{F} \subseteq 2^{N}$ is a cycle-free augmenting system with $\{i\} \in \mathcal{F}$ for all $i \in N$ if and only if $\mathcal{F}=\mathcal{F}_{L}$ for some cycle-free graph $L$.

(ii) Let $\mathcal{F} \subseteq 2^{N}$ be a normal set system. Set system $\mathcal{F}$ is an augmenting system with $\{i\} \in \mathcal{F}$ for all $i \in N$ if and only if $\mathcal{F}$ is a 2 -accessible union stable system.

\section{Cooperative games on accessible union stable sys- tems}

Let $\mathcal{F} \subseteq 2^{N}$ be a set system and let $S \subseteq N$. The maximal nonempty feasible subsets of $S$ are called components of $S$, i.e. a coalition $S \in \mathcal{F}$ is a component in $\mathcal{F}$ if there is no $T \in \mathcal{F}$ with $S \subset T$. We denote by $C_{\mathcal{F}}(S)$ the set of the components of a subset $S \subseteq N$. Observe that the set $C_{\mathcal{F}}(S)$ may be the empty set. As shown in Algaba, Bilbao, Borm and López (2000) a set system $\mathcal{F} \subseteq 2^{N}$ is union stable if and only if for any $S \subseteq N$ with $C_{\mathcal{F}}(S) \neq \emptyset$, the components of $S$ form a partition of a subset of $S$.

Definition 6.1 Let $v: 2^{N} \rightarrow \mathbb{R}$ be a cooperative game and let $\mathcal{F} \subseteq 2^{N}$ be an accessible union stable system. The restricted game $v^{\mathcal{F}}: 2^{N} \rightarrow \mathbb{R}$, is defined by

$$
v^{\mathcal{F}}(S)=\sum_{T \in C_{\mathcal{F}}(S)} v(T) \text { for all } S \subseteq N
$$

Notice that for any $S \subseteq N$ such that $C_{\mathcal{F}}(S)=\emptyset$, we have $v^{\mathcal{F}}(S)=0$. If $(N, \mathcal{F})$ is the accessible union stable system given by the connected coalitions in some graph $(N, L)$, then the game $v^{\mathcal{F}}$ is the graph-restricted game of Myerson (1977) and Owen (1986). Since an antimatroid $\mathcal{A}$ is union closed, any subset $S \subseteq N$ has a unique component given by the interior operator $\operatorname{int}(S)=\bigcup\{C \in \mathcal{A} \mid C \subseteq S\}$. The restricted game $v^{\mathcal{A}}: 2^{N} \rightarrow \mathbb{R}$, is the game defined by $v^{\mathcal{A}}(S)=v(\operatorname{int}(S))$, see Algaba, Bilbao, van den Brink and JimenezLósada (2003, 2004).

A game on an accessible union stable system is a triple $(N, v, \mathcal{F})$ where $v: 2^{N} \rightarrow \mathbb{R}$ with $v(\emptyset)=0$, and $\mathcal{F} \subseteq 2^{N}$ is an accessible union stable system. Since, again we take the player set to be fixed, we denote a game on an accessible union stable system $(N, v, \mathcal{F})$ by $(v, \mathcal{F})$. The set of all games on an accessible union stable system with player set $N$ is denoted by $G A U S^{N}$.

Next, denote by $S^{+}=S \cup a u(S)=\{i \in N \mid S \cup\{i\} \in \mathcal{F}\}$ the set $S$ together with all its augmentation points. ${ }^{11}$

\footnotetext{
${ }^{11}$ From its proof it follows that the following proposition holds for every union stable system.
} 
Proposition 6.2 Let $\mathcal{F} \subseteq 2^{N}$ be an accessible union stable system. Then the interval

$$
\left[T, T^{+}\right]_{\mathcal{F}}=\left\{S \in \mathcal{F} \mid T \subseteq S \subseteq T^{+}\right\}
$$

is a Boolean algebra for every nonempty $T \in \mathcal{F}$.

Proof: We will prove that

$$
\left\{S \in \mathcal{F} \mid T \subseteq S \subseteq T^{+}\right\}=\left\{S \subseteq N \mid T \subseteq S \subseteq T^{+}\right\}
$$

i.e., for each $S \subseteq N$ such that $T \subseteq S \subseteq T^{+}$it holds that $S \in \mathcal{F}$. We distinguish two cases.

1. If the set $a u(T)=\emptyset$ then $\left[T, T^{+}\right]_{\mathcal{F}}=\{T\}$.

2. Suppose that $a u(T) \neq \emptyset$. Since $T \subseteq S \subseteq T^{+}$, suppose without loss of generality that there exist $\left\{i_{1}, \ldots, i_{q}\right\}$ such that $a u(T)=\left\{i_{1}, \ldots, i_{q}\right\}$ and $S=T \cup\left\{i_{1}, \ldots, i_{p}\right\}$ where $1 \leq p \leq q$. In order to prove that $S \in \mathcal{F}$, we use induction on $p$. For $p=1$, $i_{1} \in a u(T)$, and therefore $T \cup\left\{i_{1}\right\} \in \mathcal{F}$. By induction, for $2 \leq k \leq q-1$, we assume that $T \cup\left\{i_{1}, \ldots, i_{k}\right\} \in \mathcal{F}$. Since $i_{k+1}$ is an augmentation point of $T$, it holds that $T \cup\left\{i_{k+1}\right\} \in \mathcal{F}$. As $\left(T \cup\left\{i_{1}, \ldots, i_{k}\right\}\right) \cap\left(T \cup\left\{i_{k+1}\right\}\right)=T \neq \emptyset$, by applying union stability we have

$$
\left(T \cup\left\{i_{1}, \ldots, i_{k}\right\}\right) \cup\left(T \cup\left\{i_{k+1}\right\}\right)=T \cup\left\{i_{1}, \ldots, i_{k}, i_{k+1}\right\} \in \mathcal{F} .
$$

An allocation rule or value for a class of games with restricted cooperation $\mathcal{C} \subseteq G A U S^{N}$ is a function $f: \mathcal{C} \rightarrow \mathbb{R}^{N}$ such that $f(v, \mathcal{F}) \in \mathbb{R}^{N}$ for all $(v, \mathcal{F}) \in \mathcal{C}$, that assigns a payoff vector to every game in this class. We consider the allocation rule that assigns to every game on an accessible union stable system the Shapley value of the corresponding restricted game.

Definition 6.3 The value $\varphi: G A U S^{N} \rightarrow \mathbb{R}^{N}$ is the allocation rule for games on accessible union stable systems defined by $\varphi(v, \mathcal{F})=\operatorname{Sh}\left(v^{\mathcal{F}}\right)$ for every $(v, \mathcal{F}) \in G A U S^{N}$, where Sh denotes the Shapley value.

For games on accessible union stable systems, the value $\varphi$ generalizes the Myerson value for games restricted by communication graphs and the (conjunctive and disjunctive) permission value for games with a permission structure.

As mentioned before, if $\mathcal{F}$ is a union stable system then the components of $N$ in $\mathcal{F}$ form a partition of a subset of $N$. (If $\mathcal{F}$ is, moreover, normal then the components form a partition of $N$ ). Component efficiency of an allocation rule on a class of games on union stable systems states that for every game with restricted cooperation in this class, the total payoff to every component equals its worth. We denote by $U S^{N}$ the class of all union stable systems on $N$, and by $G U S^{N}$ the class of all games on union stable systems on $N$. 
Definition 6.4 Let $\mathcal{C} \subseteq G U S^{N}$ be a class of games on union stable systems. An allocation rule $f$ on $\mathcal{C}$ satisfies component efficiency if $\sum_{i \in M} f_{i}(v, \mathcal{F})=v(M)$, for all $(v, \mathcal{F}) \in \mathcal{C}$ and $M \in C_{\mathcal{F}}(N)$.

A player $i \in N$ is called a component dummy in $\mathcal{F} \in U S^{N}$ if this player does not belong to any maximal component of the grand coalition, i.e., $i \notin \bigcup\left\{M \in \mathcal{F}: M \in C_{\mathcal{F}}(N)\right\}$.

Note that a component dummy in $\mathcal{F}$ is a null player in any $v^{\mathcal{F}}$ that is derived from some $v \in \mathcal{G}^{N}$.

Definition 6.5 Let $\mathcal{C} \subseteq G U S^{N}$ be a class of games on union stable systems. An allocation rule $f$ on $\mathcal{C}$ satisfies component dummy if for any component dummy in $\mathcal{F}$, we have $f_{i}(v, \mathcal{F})=0$, for all $(v, \mathcal{F}) \in \mathcal{C}$.

Algaba, Bilbao, Borm and López (2001) show that the Shapley value satisfies component efficiency and component dummy on $U S^{N}$, and therefore on every $\mathcal{C} \subseteq U S^{N}$. Next, we provide an axiomatic characterization of the Shapley value for games on the class of all accessible union stable systems that uses a balanced contribution axiom. Inspired by Myerson (1980), we will compare the effect of deleting players of the accessible union stable system on each others payoff. Given a set system $\mathcal{F} \subseteq 2^{N}$ and a player $i \in N$, the set system

$$
\mathcal{F}_{-i}=\{S \in \mathcal{F} \mid i \notin S\}
$$

is given by all those feasible coalitions in $\mathcal{F}$ which do not contain player $i$.

Proposition 6.6 If $\mathcal{F} \subseteq 2^{N}$ is an accessible union stable system and $i \in N$, then $\mathcal{F}_{-i}$ is an accessible union stable system.

Proof: If $S, T \in \mathcal{F}_{-i}$ with $S \cap T \neq \emptyset$ then by union stability $S \cup T \in \mathcal{F}$. Since $i \notin S \cup T$, it holds that $S \cup T \in \mathcal{F}_{-i}$.

Moreover, if $S \in \mathcal{F}_{-i}$ then $S \in \mathcal{F}$, and applying accessibility there exists a $j \in S$ such that $S \backslash\{j\} \in \mathcal{F}$. Since $j \neq i$, it holds that $S \backslash\{j\} \in \mathcal{F}_{-i}$.

Observe that if $\mathcal{F} \subseteq 2^{N}$ is an accessible union stable system and $i \in N$, then $i$ is a component dummy for the accessible union stable system $\mathcal{F}_{-i}$.

Definition 6.7 An allocation rule $f$ on $G A U S^{N}$ has balanced contributions if for every $(v, \mathcal{F}) \in G A U S^{N}$ and any two players $i, j \in N$ with $i \neq j$, we have

$$
f_{i}(v, \mathcal{F})-f_{i}\left(v, \mathcal{F}_{-j}\right)=f_{j}(v, \mathcal{F})-f_{j}\left(v, \mathcal{F}_{-i}\right) .
$$


Proposition 6.8 For all $(v, \mathcal{F}) \in G A U S^{N}$ and all $i, j \in N$ with $i \neq j$, the Shapley value has balanced contributions.

Proof: It is well-known that the Shapley value can be written, for every $v \in \mathcal{G}^{N}$, as

$$
S h_{i}(v)=\sum_{\{T \in \mathcal{F} \mid i \in T\}} \frac{\Delta_{v}(T)}{|T|} \text { for all } i \in N,
$$

where $\Delta_{v}(T)$ is the Harsanyi dividends of coalition $\emptyset \neq T \subseteq N$, see Harsanyi (1959). ${ }^{12}$

Consider $i \in N, j \in N \backslash\{i\}$, and denote by $\mathcal{F}_{i}=\{S \in \mathcal{F} \mid i \in S\}$ the set system formed by those feasible coalitions containing player $i$.

If $T \in \mathcal{F}_{-i}$ then every $S \in \mathcal{F}$ such that $S \subseteq T$ satisfies $i \notin S$ and hence, $S \in \mathcal{F}_{-i}$ and $S \backslash\{i\}=S$. Since $C_{\mathcal{F}_{-i}}(S)=C_{\mathcal{F}}(S \backslash\{i\})$ for all $S \subseteq N$, for $T \in \mathcal{F}_{-i}$, we have

$$
\begin{aligned}
\Delta_{v^{\mathcal{F}_{-i}}}(T) & =\sum_{S \subseteq T}(-1)^{|T|-|S|} v^{\mathcal{F}_{-i}}(S)=\sum_{S \subseteq T}(-1)^{|T|-|S|} v^{\mathcal{F}}(S \backslash\{i\}) \\
& =\sum_{S \subseteq T}(-1)^{|T|-|S|} v^{\mathcal{F}}(S)=\Delta_{v^{\mathcal{F}}}(T) .
\end{aligned}
$$

Therefore, it holds that

$$
\begin{aligned}
\varphi_{i}(v, \mathcal{F})-\varphi_{i}\left(v, \mathcal{F}_{-j}\right) & =S_{i}\left(v^{\mathcal{F}}\right)-S h_{i}\left(v^{\mathcal{F}_{-j}}\right) \\
& =\sum_{\{T \in \mathcal{F} \mid i \in T\}} \frac{\Delta_{v^{\mathcal{F}}}(T)}{|T|}-\sum_{\left\{T \in \mathcal{F}_{-j} \mid i \in T\right\}} \frac{\Delta_{v^{\mathcal{F}}-j}(T)}{|T|} \\
& =\sum_{\{T \in \mathcal{F} \mid i, j \in T\}} \frac{\Delta_{v^{\mathcal{F}}(T)}}{|T|} \\
& =\sum_{\{T \in \mathcal{F} \mid j \in T\}} \frac{\Delta_{v^{\mathcal{F}}}(T)}{|T|}-\sum_{\left\{T \in \mathcal{F}_{-i} \mid j \in T\right\}} \frac{\Delta_{v^{\mathcal{F}_{-i}}}(T)}{|T|} \\
& =S_{j}\left(v^{\mathcal{F}}\right)-S_{j}\left(v^{\mathcal{F}_{-i}}\right)=\varphi_{j}(v, \mathcal{F})-\varphi_{j}\left(v, \mathcal{F}_{-i}\right),
\end{aligned}
$$

where the third equality follows from the fact that $\Delta_{v^{\mathcal{F}}}(T)=\Delta_{v^{\mathcal{F}_{-j}}}(T)$ for all $T \in \mathcal{F}_{-j}$.

The following theorem provides a characterization of the Shapley value on the class of games on accessible union stable systems.

Theorem 6.9 The Shapley value is the unique value on the class GAUS ${ }^{N}$ that satisfies component efficiency, component dummy, and has balanced contributions.

\footnotetext{
${ }^{12}$ For each $T \subseteq N$, the unanimity game $\left(N, u_{T}\right)$ is given by $u_{T}(S)=1$ if $T \subseteq S$, and $u_{T}(S)=0$ otherwise. It is well-known that the unanimity games $u_{T}, T \subseteq N, T \neq \emptyset$, form a basis of the vectorial space of TU-games on $N$. Each game $v \in \mathcal{G}^{N}$ can be written as a linear combination of unanimity games in a unique way as $v=\sum_{T \subseteq N, T \neq \emptyset} \Delta_{v}(T) u_{T}$. By applying the Möbius transformation we obtain that $\Delta_{v}(S)=\sum_{T \subseteq S}(-1)^{|S|-|T|} v(T), S \subseteq N$.
} 
Proof: Since the Shapley value satisfies the three axioms, we only need to show uniqueness. ${ }^{13}$ Consider an allocation rule $f$ on $G A U S^{N}$ satisfying component efficiency, component dummy and has balanced contributions. We will prove that $f(v, \mathcal{F})=\varphi(v, \mathcal{F})$ for all $(v, \mathcal{F}) \in G A U S^{N}$, by induction on the number of feasible coalitions.

If $|\mathcal{F}|=1$ then $\mathcal{F}=\{\emptyset\}$ and hence every player $i \in N$ is a component dummy. By the component dummy property, $f_{i}(v, \mathcal{F})=0=\varphi_{i}(v, \mathcal{F})$ for all $i \in N$.

Consider $k>1$ and suppose that $f(v, \mathcal{F})=\varphi(v, \mathcal{F})$ for all $(v, \mathcal{F}) \in G A U S^{N}$ such that $|\mathcal{F}| \leq k-1$. Let $(v, \mathcal{F}) \in G A U S^{N}$ with $|\mathcal{F}|=k$. Observe that the grand coalition $N$ is the union of the set of disjoint dummy players and the components of $N$. Therefore, it suffices to check that for every $M \in C_{\mathcal{F}}(N)$ it holds that $f_{i}(v, \mathcal{F})=\varphi_{i}(v, \mathcal{F})$ for all $i \in M$. Let $M \in C_{\mathcal{F}}(N)$. If $|M|=1$ then component efficiency determines the payoff for $i \in M$. Otherwise, if $|M|>1$, take $i \in M$. For every $j \in M \backslash\{i\}$, we can repeatedly apply balanced contributions yielding

$$
f_{i}(v, \mathcal{F})-f_{i}\left(v, \mathcal{F}_{-j}\right)=f_{j}(v, \mathcal{F})-f_{j}\left(v, \mathcal{F}_{-i}\right), \text { for all } j \in M \backslash\{i\}
$$

This yields $|M|-1$ linear independent equations. Component efficiency requires that

$$
\sum_{i \in M} f_{i}(v, \mathcal{F})=v(M)
$$

Since the values $f\left(v, \mathcal{F}_{-i}\right)$ and $f\left(v, \mathcal{F}_{-j}\right)$ are known by the induction hypothesis, (6.3) together with the equations (6.2) yields $|M|$ linear independent equations in the $|M|$ unknown payoffs $f_{h}(\mathcal{F}, v), h \in M$, which are therefore uniquely determined.

Above, using balanced contributions, we considered the effects of deleting all coalitions containing a particular player from the set of feasible coalitions on the payoffs of another player. Comparing payoffs of two players in this way, we can also consider the effect on their payoffs when we delete all coalitions containing both players. So, for an accessible union stable system $\mathcal{F}$ and two players $i, j \in N$, we consider the set system

$$
\mathcal{F}_{-i j}=\{S \in \mathcal{F} \mid\{i, j\} \nsubseteq S\}
$$

being the collection of feasible coalitions in $\mathcal{F}$ that do not contain both players $i$ and j. Myerson (1977) characterized the Myerson value for communication graph games by component efficiency and fairness, the last property stating that deleting a link from the undirected communication graph has the same effect on the payoffs of the two players

\footnotetext{
${ }^{13}$ Note that from this proof it follows that this proposition holds for all classes of set systems such that $\mathcal{F}_{-i}$ is in the class for all $i \in N$. Although this follows from a more general result in Katsev (2013), we provided the proof for completeness and to get a better insight in the structural properties.
} 
connected by that link. This is generalized by Algaba, Bilbao, Borm and López (2001) to games on union stable systems by saying that deleting a non-unitary support from the basis of a union stable system and considering the new system generated under union stability, has the same effect on the payoff of the players in the deleted support. (Note that the supports of the set of connected coalitions in an undirected communication graph are exactly the singletons and the links.) In both cases, all coalitions that are deleted from the set of feasible coalitions contain all players in the support that is deleted. Next, we define a version of fairness where we delete all coalitions containing two particular players, and require the payoffs of these two players to change by the same amount. ${ }^{14}$

Definition 6.10 Let $\mathcal{C}$ be a class of games on union stable systems. An allocation rule $f$ on $\mathcal{C}$ satisfies fairness on $\mathcal{C}$ if

$$
f_{i}(v, \mathcal{F})-f_{i}\left(v, \mathcal{F}_{-i j}\right)=f_{j}(v, \mathcal{F})-f_{j}\left(v, \mathcal{F}_{-i j}\right)
$$

for all $(v, \mathcal{F}) \in \mathcal{C}$ and $i, j \in N$ such that $\mathcal{F}_{-i j} \in \mathcal{C}$.

The restriction that $\mathcal{F}_{-i j} \in \mathcal{C}$ implies that not all feasible coalitions can be deleted. ${ }^{15}$ It turns out that $\mathcal{F}$ being accessible implies that $\mathcal{F}_{-i j}$ is accessible.

Proposition 6.11 If $\mathcal{F} \subseteq 2^{N}$ is an accessible set system then $\mathcal{F}_{-i j}$ is accessible.

Proof: Let $\mathcal{F} \subseteq 2^{N}$ be an accessible set system. Then $S \in \mathcal{F}_{-i j}$ implies that $S \in \mathcal{F}$, and thus there is an $h \in S$ such that $S \backslash\{h\} \in \mathcal{F}$. Since $\{i, j\} \nsubseteq S \in \mathcal{F}_{-i j}$ it holds that $S \backslash\{h\} \in \mathcal{F}_{-i j}$. So, $\mathcal{F}_{-i j}$ is accessible.

However, for an arbitrary accessible union stable system $\mathcal{F}$ the set system $\mathcal{F}_{-i j}$ need not be union stable as the following example shows.

Example 6.12 Consider the accessible union stable system $\mathcal{F}$ of Example 3.6. Take a player from $N$ and one from $M$, for example players 2 and 4.

Then $\mathcal{F}_{-24}=\mathcal{F} \backslash\{\{2,3,4,5\},\{1,2,3,4,5\}\}$, which is not union stable since $\{1,2\}$ and $\{1,3,4,5\}$ both belong to $\mathcal{F}_{-24}$ but their union does not.

\footnotetext{
${ }^{14}$ Note that if $\mathcal{F}=\mathcal{F}_{L}$ for some communication graph $L$ then $\mathcal{F}_{-i j}$, in general, is not the set of connected coalitions in $L \backslash\{\{i, j\}\}$. Consider, for example, the communication graph $L$ on $N=\{1,2,3,4\}$ given by $L=\{\{1,2\},\{2,3\},\{3,4\},\{1,4\}\}$. Then $\{1,2,3,4\} \in \mathcal{F}_{L \backslash\{\{1,2\}\}} \backslash\left(\mathcal{F}_{L}\right)_{-12}$. Therefore, on the class of communication graph games this axiom is not the same as Myerson's fairness.

${ }^{15} \mathrm{So}$, we should call this axiom fairness with respect to a certain class of games with restricted cooperation. When it does not lead to confusion we will simply refer to this axiom as fairness.
} 
Before we mentioned that fairness as defined in Definition 6.10 for the class of accessible union stable systems is not the same as Myerson's fairness. However, for (sets of connected coalitions in) cycle-free graphs it is the same. Therefore, it is known that for these set systems, fairness and component efficiency characterize the Shapley value. Similar it follows that for the class games on cycle-free accessible union stable systems, the Shapley value is characterized by component efficiency, component dummy and fairness. We give an alternative proof considering this as a subclass of games on acccessible union stable systems and applying the results obtained for cycle-free accessible union stable systems resulting in a much better understanding of these structures.

Next, we denote the class of games on cycle-free accessible union stable systems by $G C F^{N}$. It turns out that union stability of the set system $\mathcal{F}_{-i j}$ is kept if the accessible union stable system belongs to $C F^{N}=A U S^{N} \cap U S I^{N}$ and players $i$ and $j$ belong to a support together.

Proposition 6.13 If $\mathcal{F} \in C F^{N}$ and $\{i, j\} \in B(\mathcal{F})$, then $\mathcal{F}_{-i j}$ is union stable.

Proof: Suppose that (i) and (ii) are satisfied. Take $S, T \in \mathcal{F}_{-i j}$ with $S \cap T \neq \emptyset$. We have to prove that $S \cup T \in \mathcal{F}_{-i j}$. We distinguish the following two cases.

Case 1: Suppose that $\{i, j\} \nsubseteq S \cup T$. Since $S \cup T \in \mathcal{F}$ (by union stability of $\mathcal{F}$ ) it holds that $S \cup T \in \mathcal{F}_{-i j}$.

Case 2: Suppose that $\{i, j\} \subseteq S \cup T$. Since $S, T \in \mathcal{F}_{-i j}$ it holds that $|S \cap\{i, j\}|=$ $|T \cap\{i, j\}|=1$ with $S \cap\{i, j\} \neq T \cap\{i, j\}$. Suppose without loss of generality that $i \in S$ and $j \in T$. Since there is an $H \in B(\mathcal{F})$ with $\{i, j\} \subseteq H,|H \cap(S \cup T)| \geq 2$. Since $\mathcal{F}$ satisfies condition 1 of Definition 5.1 (2-intersection closedness), it holds that $H \cap(S \cup T) \in \mathcal{F}$. But then $(H \cap(S \cup T)) \cup(S \cup T)=S \cup T, H \cap(S \cup T) \neq S$ and $H \cap(S \cup T) \neq T$ (since $\{i, j\} \subseteq H,\{i, j\} \subseteq S \cup T, i \notin T$ and $j \notin S)$. This is a contradiction with $\mathcal{F} \in C F^{N}$ since $S \cup T$ can be written as union of non-unitary supports in more than one way.

When we restrict ourselves to the class $C F^{N}$ we also need to verify if deleting all coalitions that contain two particular players, we still have a set system from $C F^{N}$.

Proposition 6.14 If $\mathcal{F} \in C F^{N}$ and $\{i, j\} \in B(\mathcal{F})$ then $\mathcal{F}_{-i j} \in C F^{N}$.

Proof From Propositions 6.11, 6.13 and Corollary ?? it follows that we only need to verify the extra condition 2 of Definition 5.1 defining the class $C F^{N}$

Since every $S \in \mathcal{F}$ can be written as a union of non-unitary supports in a unique way, this obviously holds for every $S \in \mathcal{F}^{\prime} \subseteq \mathcal{F}$, in particular for $\mathcal{F}^{\prime}=\mathcal{F}_{-i j}$.

Now we can state the following axiomatization. 
Theorem 6.15 The Shapley value is the unique value on $G C F^{N}$ that satisfies component efficiency, component dummy and fairness.

Proof The Shapley value satisfying component efficiency, component dummy and fairness can be shown in a similar way as shown in Algaba, Bilbao, Borm and López (2001). To show uniqueness, suppose that an allocation rule $f$ satisfies component efficiency, component dummy and fairness. We prove uniqueness (in a similar way to Myerson (1977) and Algaba, Bilbao, Borm and López (2001) but) by induction on the number of nonunitary coalitions in $\mathcal{F}$. If $|\{S \in \mathcal{F}|| S \mid \geq 2\}|=0$ then every singleton is a component or a dummy, and then component efficiency or component dummy, respectively, determines the payoffs. Proceeding by induction, assume that $f(v, \mathcal{F})$ is determined whenever $|\{S \in \mathcal{F}|| S \mid \geq 2\}|<k$, and suppose that $|\{S \in \mathcal{F}|| S \mid \geq 2\}|=k$. Observe that the grand coalition $N$ is the union of the set of disjoint dummy players and the components of $N$. Therefore, it suffices to check that for every $M \in C_{\mathcal{F}}(N)$ it holds that $f_{i}(v, \mathcal{F})=\varphi_{i}(v, \mathcal{F})$ for all $i \in M$. Let $M \in C_{\mathcal{F}}(N)$. If $|M|=1$ then component efficiency determines the payoff for $i \in M$. Otherwise, if $|M|>1$, we know from Algaba, Bilbao, Borm and López (2001) that we can label the players in $M=\left\{i_{1}, \ldots, i_{m}\right\}$ such that for every $k \in\{1, \ldots, m-1\}$ there is a support $H=\left\{i_{k}, i_{k+1}\right\}$. Since $\mathcal{F}_{-i_{k} i_{k+1}} \in C F^{N}$ by Proposition 6.14 , we can repeatedly apply fairness yielding

$$
f_{i_{k}}(v, \mathcal{F})-f_{i_{k}}\left(v, \mathcal{F}_{-i_{k} i_{k+1}}\right)=f_{i_{k+1}}(v, \mathcal{F})-f_{i_{k+1}}\left(v, \mathcal{F}_{-i_{k} i_{k+1}}\right), k \in\{1, \ldots, m-1\}
$$

This yields $|M|-1$ linear independent equations. Component efficency requires that

$$
\sum_{i \in M} f_{i}(v, \mathcal{F})=v(M)
$$

Since the values $f\left(N, v, \mathcal{F}_{-i_{k} i_{k+1}}\right)$ are known by the induction hypothesis, together with the equations (6.4) this yields $|M|$ linear independent equations in the $|M|$ unknown payoffs $f_{h}(N, \mathcal{F}, v), h \in M$, which are therefore uniquely determined.

\section{Concluding remarks}

Some future research questions are the following. First, there are several models of restricted cooperation in the literature. It is important to compare them and see the relations between these models: What can they 'learn' from each other?

When deleting coalitions from a set of feasible coalitions that satisfies certain properties we need to take care that the resulting set of feasible coalitions still satisfies these 
properties. For example, in a union stable system only supports can be deleted (since other coalitions are the union of two nondisjoint coalitions and deleting them violates union stability) while for antimatroids only paths (since otherwise union closedness is violated) that are not covered by a path ${ }^{16}$ can be deleted (since deleting a coalition that is covered by a path violates accessibility). Note that when coalition $S \in \mathcal{F}$ is covered by $T \in \mathcal{F}$ then there is an $i \in T$ such that $T$ is an $i$-path and $S=T \backslash\{i\}$. Algaba, Bilbao, Borm and López (2001) showed that Myerson (1977)'s axiomatization for communication graph games by component efficiency and fairness ${ }^{17}$ also holds for games on union stable systems, i.e. the Shapley value is the unique allocation rule for games on union stable systems that satisfies component efficiency, component dummy and fairness. The fact that not all supports can be deleted from an accessible union stable system creates problems when axiomatizating the Shapley value for games on these systems. For example, van den Brink (1997) and Algaba, Bilbao, van den Brink and Jimenez-Lósada (2003) show that such axioms do not characterize the Shapley value for games with a permission structure, respectively games on (normal) antimatroids. A main question then is whether the Shapley value for accessible union stable systems is characterized by component efficiency, component dummy and fairness. This is an important question since our motivation to study accessible union stable systems is that they have a characterizing property from communication networks (union stability) as well as hierarchies (accessibility).

A related question is on what union stable systems the Shapley value is characterized by component efficiency and fairness (of course, fairness defined with respect to the appropriate class of set systems). Characterizing set systems by axiomatizations of game solutions is done in, e.g., Algaba, Bilbao, van den Brink and Jiménez-Losada (2003) for the class of games on antimatroids. They show that on a class of antimatroids the Shapley value is characterized by efficiency, additivity, the inessential player property, the necessary player property and structural monotonicity, if and only if this is the class of poset antimatroids.

Finally, in the network formation and stability model of Jackson and Wolinsky (1996), worths are not assigned to coalitions of players, but to the network structures themselves. For example, in the two network structures $L=\{\{1,2\},\{1,3\}\}$ respectively $L^{\prime}=L \cup\{\{2,3\}\}$ on $N=\{1,2,3\}$ (see Figure 3) the 'grand coalition' $N$ can have a different value, although in a restricted game in the sense of Myerson (1977) coalition $N$ should have the same worth in both restricted games. Since in this paper we studied more rich network structures than the bilateral communication networks, it makes sense to consider network value functions similar to those in Jackson and Wolinsky (1996), only now for accessible

\footnotetext{
${ }^{16}$ Coalition $S \in \mathcal{F}$ is covered by a path if there is a path $T \in \mathcal{F}$ such that $S \subset T$ and $|T|=|S|+1$.

${ }^{17}$ For communication graph games component dummy is not relevant since every singleton is feasible and thus there are no dummies in the set of connected coalitions.
} 


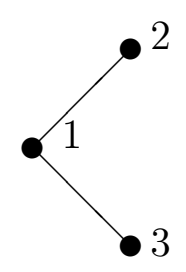

Figure 3: Two connected communication graphs on $N=\{1,2,3\}$

union stable systems and other structures.

\section{References}

Algaba E., Bilbao J.M., Borm P, López J.J. (2000), "The position value for union stable systems", Mathematical Methods of Operations Research, 52, 221-236.

Algaba E., Bilbao J.M., Borm P, López J.J. (2001), "The Myerson value for union stable structures", Mathematical Methods of Operations Research, 54, 359-371.

Algaba, E., J.M. Bilbao, R. van den Brink, And A. Jiménez-Losada (2003), "Axiomatizations of the Shapley value for cooperative games on antimatroids", Mathematical Methods of Operations Research , 57, 49-65.

Algaba, E., J.M. Bilbao, R. van den Brink, And A. Jiménez-Losada (2004a), "Cooperative games on antimatroids," Discrete Mathematics, 282, 1-15

Algaba, E., J.M. Bilbao, And M. Slikker (2010), "A value for games restricted by augmenting systems", SIAM Journal on Discrete Mathematics, 24, 9921010.

Ambec, S., And Y. Sprumont (2002), "Sharing a river", Journal of Economic Theory 107, 453-462.

Bilbao, J.M. (2000) Cooperative Games on Combinatorial Structures, Kluwer Academic Publishers.

BilbaO, J.M. (2003) "Cooperative games under augmenting systems", SIAM Journal on Descrete Mathematics 17, 122-133.

Borm P., G. Owen, And S. TiJs (1992), "On the Position Value for Communication Situations", SIAM Journal on Discrete Mathematics, 5, 305-320. 
Brink, R. VAN DEN (1997), "An Axiomatization of the Disjunctive Permission Value for Games with a Permission Structure", International Journal of Game Theory, 26, $27-43$.

BRINK, R. VAN DEN (2012), "On hierarchies and communication", Social Choice and Welfare, 39, 721-735.

BRINK R. VAN DEN, AND R.P. GILles (1996), "Axiomatizations of the Conjunctive Permission Value for Games with Permission Structures", Games and Economic Behavior, $12,113-126$.

Curiel, I., J. Potters, V. Rajendra Prasad, S. Tijs and B. Veltman (1993), "Cooperation in one machine scheduling", ZOR: Methods and Models of Operations Research, 38, 113-129.

Curiel, I., J. Potters, V. Rajendra Prasad, S. Tijs and B. Veltman (1994), "Sequencing and cooperation", Operations Research, 54, 323-334.

Demange, G. (1994), "Intermediate preferences and stable coalition structures", Journal of Mathematical Economics, 23, 45-58.

Demange, G. (2004), "On group stability in hierarchies and networks", Journal of Political Economy, 112, 754-778.

Dilworth, R. P. (1940) "Lattices with Unique Irreducible Decompositions," Annals of Mathematics, 41, 771-777.

Edelman, P. H., And Jamison, R. E. (1985) "The Theory of Convex Geometries," Geometrica Dedicata, 19, 247-270.

Gilles, R. P., And Owen, G. (1994), Cooperative games and disjunctive permission structures, Department of Economics, Virginia Polytechnic Institute and State University, Blacksburg, Virginia.

Gilles R.P., G. OWen, AND R. VAn den Brink (1992), "Games with Permission Structures: The Conjunctive Approach", International Journal of Game Theory, 20, 277293.

Graham, D.A., Marshall, R.C., Richard, J.F. (1990), "Differential payments within a bidder coalition and the Shapley value", American Economic Review, 80, 493-510.

Hamiache, G. (1999), "A Value with Incomplete Communication", Games and Economic Behavior, 26, 59-78. 
Harsanyi, J.C. (1959), "A Bargaining Model for Cooperative n-Person Games", In Contributions to the Theory of Games IV (eds. Tucker A.W., and R.D. Luce), Princeton UP, Princeton pp 325-355.

JACKSOn, M.O., AND A. Wolinsky (1996), "A strategic model of social and economic networks", Journal of Economic Theory, 71, 44-74.

Jackson, M.O., T. Rodriguez-Barraquer And X. TAn (2012), "Social Capital and Social Quilts: Network Patterns of Favor Exchange", American Economic Review, 102, 1857-97.

Kaneko, M., And M. Wooders (1982), "Cores of Partitioning Games", Mathematical Social Sciences, 3, 313-327.

KATSEv, I. (2013), "The Shapley value for games with restricted cooperation", working paper, St.Petersburg Institute for Economics and Mathematics.

Korte, B., L. Lovász, And R. Schrader (1991), Greedoids, Springer, Berlin.

Le Breton, M., G. Owen And S. Weber (1992), "Strongly balanced cooperative games", International Journal of Game Theory, 20, 419-427.

Littlechild, S.C, And G. Owen (1973), "A simple expression for the Shapley value in a special case", Management Science, 20, 370-372.

Myerson, R.B. (1977), "Graphs and Cooperation in Games", Mathematics of Operations Research, 2, 225-229.

Myerson, R.B. (1980), "Conference structures and fair allocation rules", International Journal of Game Theory 9, 169-182.

Ni, D., AND Y. WANG (2007), "Sharing a polluted river", Games and Economic Behavior 60, 176-186.

Owen, G. (1986), "Values of Graph-Restricted Games", SIAM Journal of Algebraic Discrete Methods, 7, 210-220.

Shapley, L.S. (1953) "A Value for n-Person Games", In Annals of Mathematics Studies 28 (Contributions to the Theory of Games Vol.2) (eds. Kuhn H.W., and A.W. Tucker), Princeton UP, Princeton pp 307-317. 